\title{
Far-Red Spectroscopy of Peculiar Stars and the GAIA Mission
}

\author{
Ulisse Munari \\ Osservatorio Astronomico di Padova, Sede di Asiago, 36012 Asiago \\ (VI), Italy
}

\begin{abstract}
The coming GAIA Cornerstone mission by ESA will provide micro-arcsec astrometry, $\sim 10$ bands photometry and far-red spectroscopy for a huge number of stars in the Galaxy $\left(10^{9}\right)$. GAIA spectroscopy will cover the range 8480-8740 Åwhich includes the CaII triplet and the head of the Paschen series. In this paper we address the diagnostic potential of this wavelength range toward detection of peculiar stars.
\end{abstract}

\section{Introduction}

The large impact that the Hipparcos astrometric mission by ESA had on many fields of astrophysics is well known to the community. This is even more remarkable considering that the Hipparcos observations were complete to just $V \approx 8$ mag and the horizon for astrometric errors less than $10 \%\left(R_{10 \%}\right)$ was limited to $0.1 \mathrm{kpc}$, i.e. the solar neighborhood. Nevertheless, Hipparcos data have been the main driver or at least a contributor to more than 1900 papers since the release to the community of the mission data in 1997.

Hipparcos was still flying when the European community began openly speaking of its successor, with ideas already well in focus about GAIA by the time of the Cambridge 1995 ESA colloquium on the future of astrometry in space (ESA SP-379). Since then, GAIA has been at the center of a continentwide effort dealing with its science goals and the technical design, culminated with the formal mission approval in the fall of 2000. Perryman et al. (2001, and references therein) provides a useful introduction to GAIA.

Before GAIA (http://astro.estec.esa.nl/GAIA/), with a launch scheduled for not later than 2012, two other survey astrometric missions could fly if their current financial problems are eventually overcome (cf. Table 1), the German DIVA (http://www.ari.uni-heidelberg.de/diva/) and the USA mission FAME (http://www.usno.navy.mil/FAME/), both with astrometric goals intermediate between those of Hipparcos and GAIA. Finally, the technological demonstrator for interferometry in space SIM (http://sim.jpl.nasa.gov/), base-lined by NASA for a launch in 2009 , will obtain micro-arcsec astrometry of a preselected limited sample of objects. While all satellites will perform photometry in parallel with astrometry, only GAIA will also collect spectra with the main aim of deriving radial velocities and therefore to measure the $6^{\text {th }}$ component of the phase-space (the other five being provided by astrometry). 
Table 1. Comparison between the performances of Hipparcos, DIVA, FAME, SIM and GAIA astrometric missions (courtesy F. Mignard).

\begin{tabular}{lccccc}
\hline & Hipparcos & DIVA & FAME & SIM & GAIA \\
\hline Mission Type & scanning & scanning & scanning & pointing & scanning \\
Input Catalogue & Yes & No & Yes & Yes & No \\
V $_{\text {completness }} /$ mag & 8 & 12.5 & 14 & - & 20 \\
No. of objects & 118,218 & $3 \cdot 10^{7}$ & $4 \cdot 10^{7}$ & 20,000 & $1 \cdot 10^{9}$ \\
$\sigma_{\pi} / \mu$ as & $1000(V=9)$ & $200(V=9)$ & $50_{(V=9)}$ & $4(V=16)$ & $10_{(V=15)}$ \\
$\mathrm{R}_{10 \%} / \mathrm{kpc}$ & 0.1 & 0.5 & 2 & 25 & 10 \\
Spectra and RV & No & No & No & No & Yes \\
\hline \hline
\end{tabular}

\section{GAIA spectroscopy}

GAIA will survey the whole sky by spinning every three hours around an axis that in turn will precess around the Sun. For a given star during a single spin rotation, GAIA will collect one astrometric measurement, one spectrum and one photometric reading in each of $\sim 10$ bands. During the planned 5-year mission lifetime, while scanning the sky GAIA will visit each star about 100 times. Therefore about 100 astrometric positions, 100 spectra and 100 readings in each photometric band will be recorded for each target star during the mission life-time.

The stars will cross the GAIA field of view for a time interval fixed by the satellite spin rate, and this sets the equivalent exposure time for the CCDs on the focal plane (which are read in TDI mode), i.e. an exposure time equal for all stars independent of their magnitude. Assuming a resolving power of $\sim 10,000$ and a dispersion of $0.5 \AA /$ pix for GAIA spectra (still to be finalized), and the current optical design and efficiencies, Table 2 lists the magnitude of stars producing spectra of $\mathrm{S} / \mathrm{N}=100,30$ and 10 .

GAIA will record spectra over the 8480 - 8740 Årange, covering the CaII triplet, the head of the Paschen series, NI multiplets \#1 and \#8, many lines of FeI, TiI, MgI, SiI, MnI, SI and the CN and TiO molecules. Fig. 1 shows the progression of spectral changes in the GAIA wavelength region along the MKK classification system for normal stars. The classification potential is excellent, particularly for cool stars ( $\mathrm{G}-\mathrm{K}$ types being the dominant population among field stars at the faint magnitudes reached by GAIA). A general introduction to GAIA spectroscopy is provided by Munari (1999) and Munari (2002), an evaluation of GAIA performances on eclipsing binaries is given by Munari et al. (2001) and Zwitter (page 31), and the accuracy of GAIA radial velocities has been investigated by Munari, Agnolin, \& Tomasella (2001), Katz et al. (2002) and Zwitter (2002).

Figs. 2 and 3 present a sample of spectra of peculiar and exotic objects, extracted from a much larger survey performed with the Echelle+CCD spectrograph at the $1.82 \mathrm{~m}$ telescope in Asiago, operated in GAIA mode (Munari et al. 2002, A\&A, to be submitted).

The various types of peculiar stars are well distinguished among themselves and with respect to normal stars by the presence of emission lines of different 


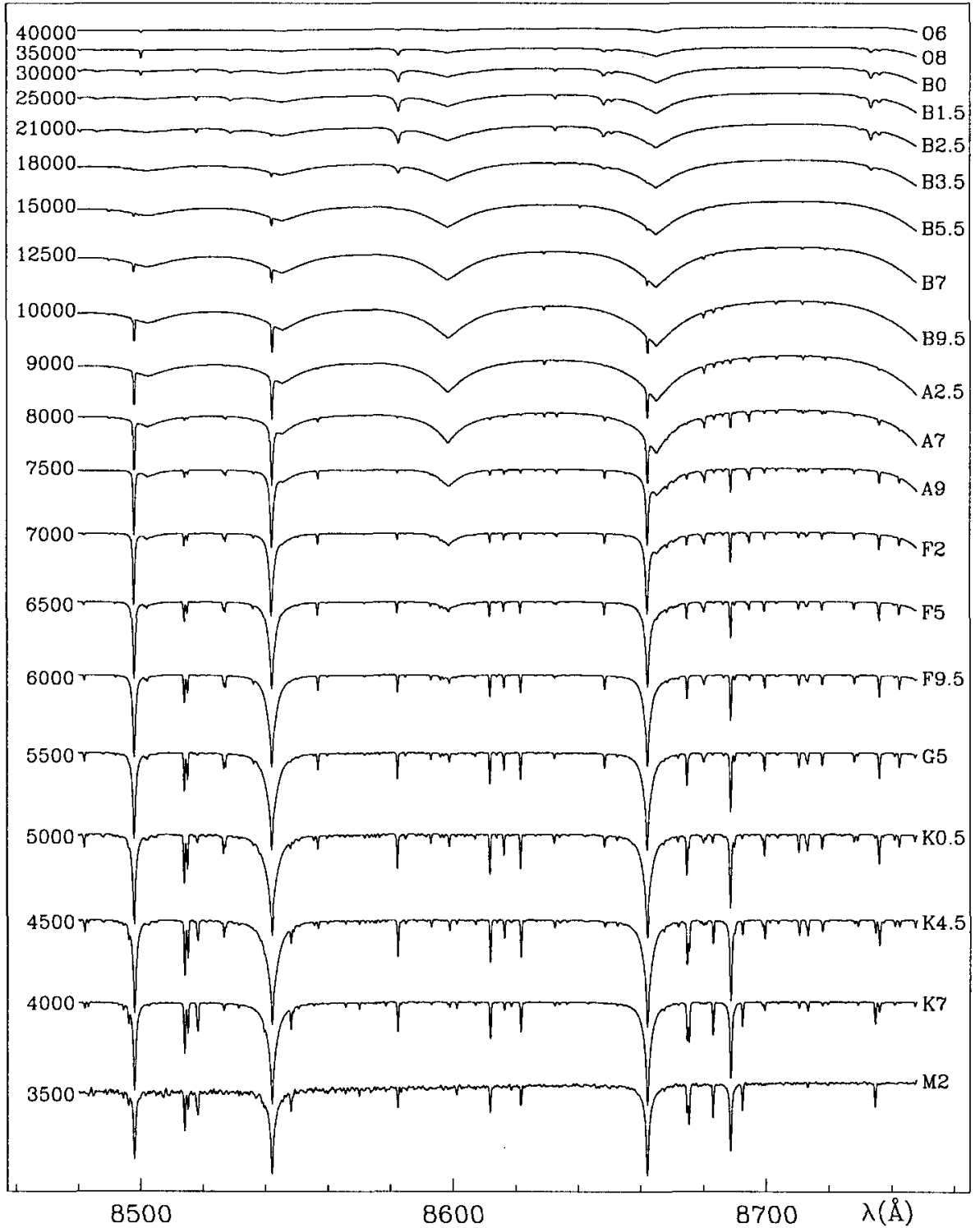

Figure 1. Sequence of synthetic spectra (from Munari \& Castelli 2000 , Castelli \& Munari 2001) illustrating the variations along the main sequence ( $T_{\text {eff }}$ in $\mathrm{K}$ on the left and corresponding spectral type for mainsequence stars on the right) for moderately metal poor stars $\left(\left[Z / Z_{\odot}\right]=\right.$ -0.5). All spectra are on the same ordinate scale, only displaced in their zero-points. A detailed mapping of the MKK classification system with real spectra is provided in the atlas by Munari \& Tomasella (1999). 


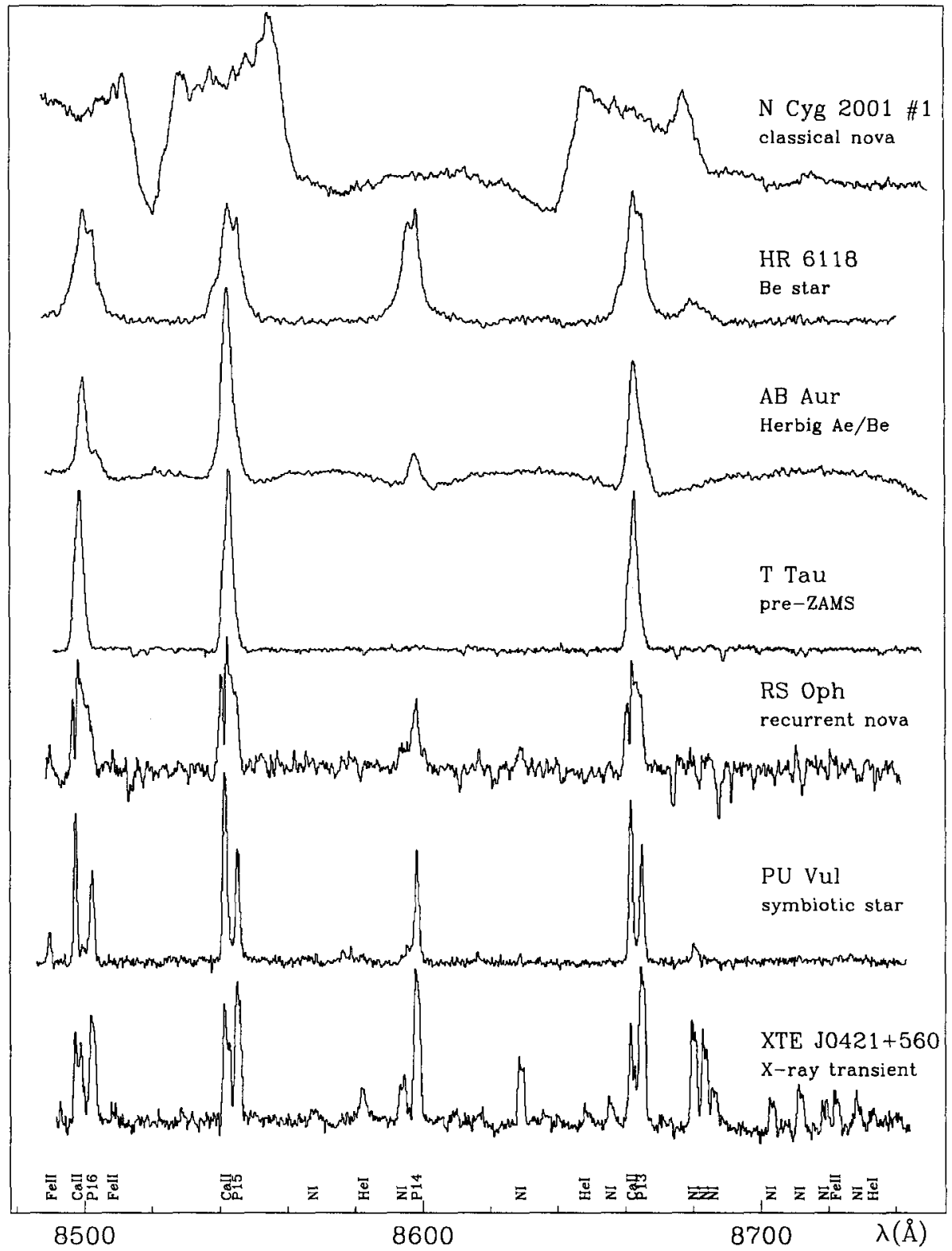

Figure 2. Examples of far-red spectra of peculiar objects dominated by emission lines (see identification at bottom). Spectra obtained with the Asiago Echelle+CCD spectrograph. The expected GAIA spectra should resemble those shown here. 


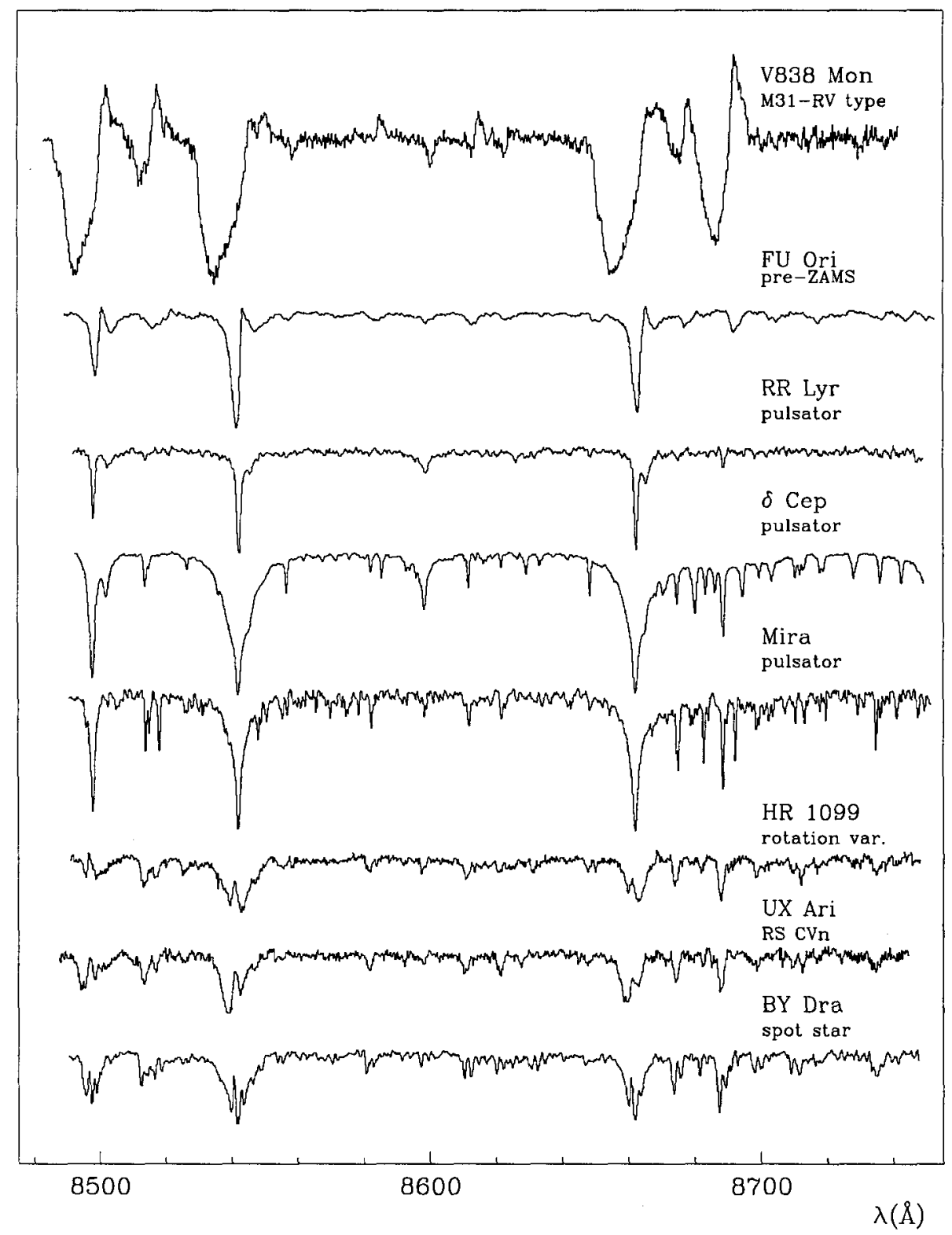

Figure 3. Additional examples of far-red spectra of peculiar objects, this time dominated by absorptions (mainly from CaII, FeI, TiI, MgI, SiI, MnI, SI, CN and TiO). Note the P-Cyg CaII profiles in V838 Mon and FU Ori, and the emission components within the CaII absorption profiles for the stars with active photospheres (HR 1099, UX Ari and BY Dra). Spectra of prototype pulsating stars are also shown. 
Table 2. The table provides the magnitudes of the stars that produce GAIA spectra with $\mathrm{S} / \mathrm{N}=100,30$ or 10 (per pixel, at $0.5 \AA / \mathrm{pix}$ dispersion and 10,000 resolving power) per single passage (values to the left) and mission-averaged (values to the right). The magnitudes are computed for the Cousins' $I$ band, which covers the wavelength range of GAIA spectra. The corresponding $V$ magnitudes are listed for F5 $\left(V-I_{\mathrm{C}}=+0.51\right)$, G5 $\left(V-I_{\mathrm{C}}=+0.73\right)$ and $\mathrm{K} 5\left(V-I_{\mathrm{C}}=+1.34\right)$ unreddened main-sequence stars $(\mathrm{G}-\mathrm{K}$ stars are the dominant spectral types of field stars at the faint magnitudes reached by GAIA).

\begin{tabular}{r|rrrr|cccc}
\hline $\mathrm{S} / \mathrm{N}$ & \multicolumn{1}{|c}{$I$} & $V_{\mathrm{F} 5 \mathrm{~V}}$ & $V_{\mathrm{G} 5 \mathrm{~V}}$ & $V_{\mathrm{K} 5 \mathrm{~V}}$ & $I$ & $V_{\mathrm{F} 5 \mathrm{~V}}$ & $V_{\mathrm{G} 5 \mathrm{~V}}$ & $V_{\mathrm{K} 5 \mathrm{~V}}$ \\
\hline 100 & 8.66 & 9.17 & 9.39 & 10.00 & 13.56 & 14.07 & 14.29 & 14.90 \\
30 & 11.27 & 11.78 & 12.00 & 12.61 & 16.07 & 16.58 & 16.80 & 17.41 \\
10 & 13.51 & 14.02 & 14.24 & 14.85 & 18.21 & 18.72 & 18.94 & 19.55 \\
\hline \hline
\end{tabular}

species and intensity, and characterized by profiles of different shape and width. In cool peculiar stars the emission in the CaII dominates (cf. T Tau), and as the temperature rises Paschen lines grow in strength while CaII decreases (cf. AB Aur). As the temperature further increases (cf. HR 6118) CaII vanishes and Paschen lines reach peak intensities. Objects with highly stratified and distinct emission regions (cf. PU Vul and XTE J0421+560) present a wide range of emission lines characterized by quite different excitation conditions, including CaII, Paschen, HeI, NI and FeII. Fast expansion and ejecta clumpiness in novae (cf. Nova Cyg 2001 \#1) is well traced in the CaII profiles close to maximum and by Paschen lines at later phases, while conspicuous mass loss (cf. V838 Mon) manifests itself in wide $\mathrm{P}$-Cyg profiles. The presence of active regions on the surface of cool stars is revealed by emission components within the CaII absorption profiles (cf. HR 1099, UX Ari and BY Dra), which change in position and intensity traces the rotation of the stars and the life-time of the hot spots.

\section{References}

Castelli, F., \& Munari, U. 2001, A\&A 366, 1003

Katz, D., Viala, Y., Gomez, A., \& Morin, D. 2002, in GAIA: A European Space Project, ed. O. Bienaymé \& C. Turon (Paris: Editions de Physique), 64 Munari, U. 1999, in ESA GAIA Workshop, ed. V. Strayzis, Balt. Astron., 8, 73 Munari, U. 2002, in GAIA: A European Space Project, ed. O. Bienaymé \& C. Turon, (Paris: Editions de Physique), 39

Munari, U., Agnolin, P., \& Tomasella, L. 2001, Balt. Astron. 10, 613

Munari, U., \& Castelli F. 2000, A\&AS 141, 141

Munari, U., \& Tomasella L. 1999, A\&AS 137, 521

Munari, U., et al. 2001, A\&A, 378, 477

Perryman, M. A. C., et al. 2001, A\&A 369, 339

Zwitter, T. 2002, A\&A 386, 748 